\title{
Interference Experiment with a Transparent Mask Rejects Wave Models of Light
}

\author{
John C. Hodge \\ Franklin, NC, USA \\ Email: jchodge@frontier.com
}

How to cite this paper: Hodge, J.C. (2019) Interference Experiment with a Transparent Mask Rejects Wave Models of Light. Optics and Photonics Journal, 9, 75-80. https://doi.org/10.4236/opj.2019.96008

Received: April 28, 2019

Accepted: June 15, 2019

Published: June 18, 2019

Copyright $\odot 2019$ by author(s) and Scientific Research Publishing Inc. This work is licensed under the Creative Commons Attribution International License (CC BY 4.0).

http://creativecommons.org/licenses/by/4.0/

\begin{abstract}
Young's double-slit experiment shows characteristics of light that are modeled as indicating a wave nature of light. Other experiments suggest a particle model of light. An experiment is performed with the Fraunhofer pattern from a first mask impinging on a transparent second mask with a slit. The screen pattern is an interference pattern such as produced in Young's Experiment. An opaque strip between the first and second mask blocks the light of the center maxima from the first mask. The screen interference fringes remained. Moving the glass mask so the slit is removed from light shows the necessity of a slit in the second mask. This suggests a Newtonian type of light model. This experiment rejects the wave models of light.
\end{abstract}

\section{Keywords}

Diffraction, Interference, Young's Experiment, Light, Photon

\section{Introduction}

A single model of light has remained a mystery. Some experiments such as the photoelectric effect support the traditional particle model of light. Other experiments such as diffraction and interference support the traditional wave models of light.

Newton speculated light is a stream (ray) of corpuscles [1]. A wave in the aether travels faster than the corpuscles and directs the corpuscles' path. Newton thought the effect light produces as two different entities like a rock (photon) creating waves in water (aether).

The prevailing models of the 19th century started with Young's experiment and considered light to be a wave. Several wave models have been developed that describe the interference pattern of light on the screen such as Fraunhofer, Fresnel, Sommerfield and Kirchhoff ([2], p. 379). Wave models depended on the 
Huygens-Fresnel principle that stated each point along the wave crest is a source of a new, spherical wave. The diffracting waves are assumed to originate in and be limited by the slit. The slit limitation is crucial in the wave model. It allows the waves through the slit to expand sideways. The waves from each point across the slit interfere with other waves to form the diffraction pattern. Single slit model's intensity distributions on a screen are described by the Fraunhofer equation farther from the slit ([2], Section 18.17).

Young's double-slit experiment is an example of the interference of light. The diffraction peaks from a single-slit appear superimposed on light from a second slit to produce multiple fringes for each diffraction peak. Opaque strips in coherent light also give diffraction effects that are ripples in the pattern. The distinction between fringes and ripples is that the fringe minima are at or near zero intensity and the ripples are small variations in intensity without going to zero unless the impinging light has low amplitude.

A model of redshift and discrete redshift used a photon model to explain the observations [3]. This model was extended to suggest this experiment.

This paper has the diffraction pattern from the first mask impinge on a transparent, second mask with a slit. The description of the experiment is in Section 2. The discussion and conclusion are in Section 3.

\section{The Experiment}

The diagram in Figure 1 shows the experimental setup.

The $5 \mathrm{~mW}, 635 \mathrm{~nm}$ laser was manufactured to be a pointer. The first mask was $15 \mathrm{~cm}$ from the laser. The first mask slit was $0.5 \mathrm{~mm}$ wide. The second mask was approximately $240 \mathrm{~cm}$ from the first mask, was a $23 \mathrm{~mm}$ thick windowpane glass plate, and had a $1.5 \mathrm{~mm}$ wide slit. The second mask was placed such that the width of the slit was approximately half the width of the central peak from the first mask as depicted in Figure 2. The screen was $6.6 \mathrm{~m}$ from the first mask.

Figure 3 shows photographs of the images on the screen at each noted stage of the experiment. The images in the photographs are actually red and have been converted to gray shades for printing. Figure 3(a) is the image of the diffraction pattern from the first mask. It is a Fraunhofer diffraction pattern. Figure 3(b) is the image of the diffraction pattern from the first mask with transparent glass without a slit as a second mask. Figure $3(\mathrm{c})$ is the image of the screen pattern when a slit in the glass of the second mask centered on the maxima of the major peak from the first mask as noted in Figure 2. Note the interference fringes. Figure $3(c)$ is a typical interference pattern.

When an opaque strip (nail in this experiment) is placed as a second mask in the middle of the primary peak from the first mask, an interference pattern is projected onto the screen (see Figure 4(left). Note the interference ripples in the shadow of the nail. The secondary diffraction peaks have ripples as the effect of an edge of the nail (see Figure 5(top), see [2], p. 376).

However, the pattern differs when the edges of the nail are in the minima of 


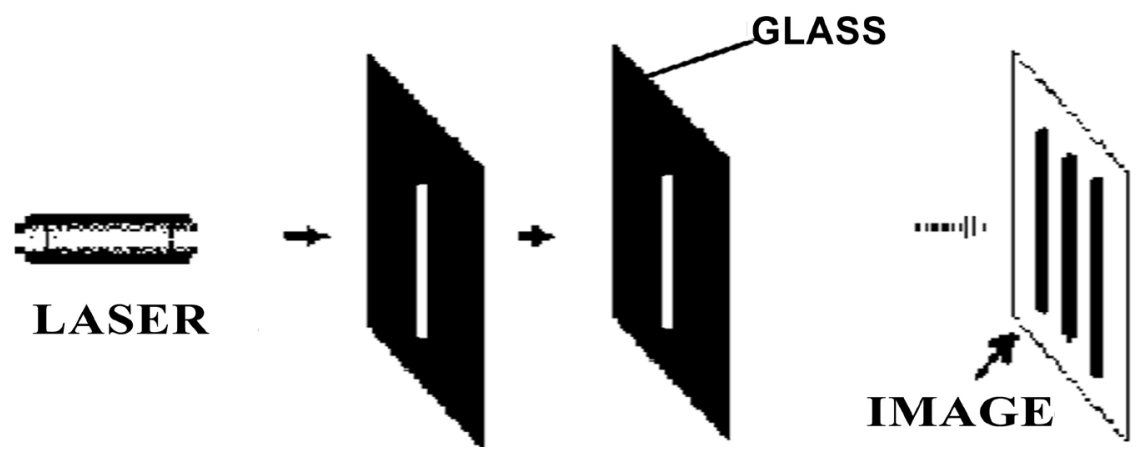

Figure 1. Diagram of the experimental fixtures.

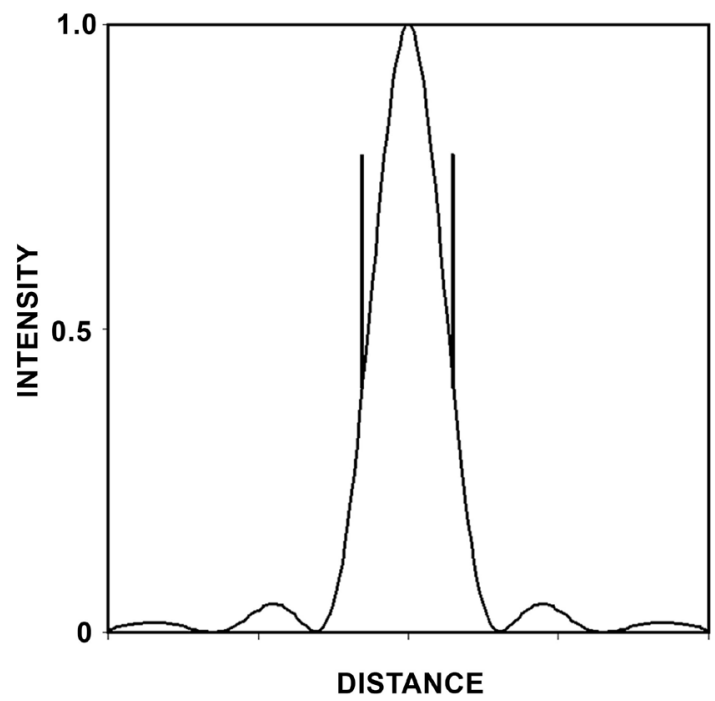

Figure 2. The Fraunhofer diffraction pattern that impinges onto the second mask. The straight lines mark the edges of the second mask slit relative to the diffraction pattern.

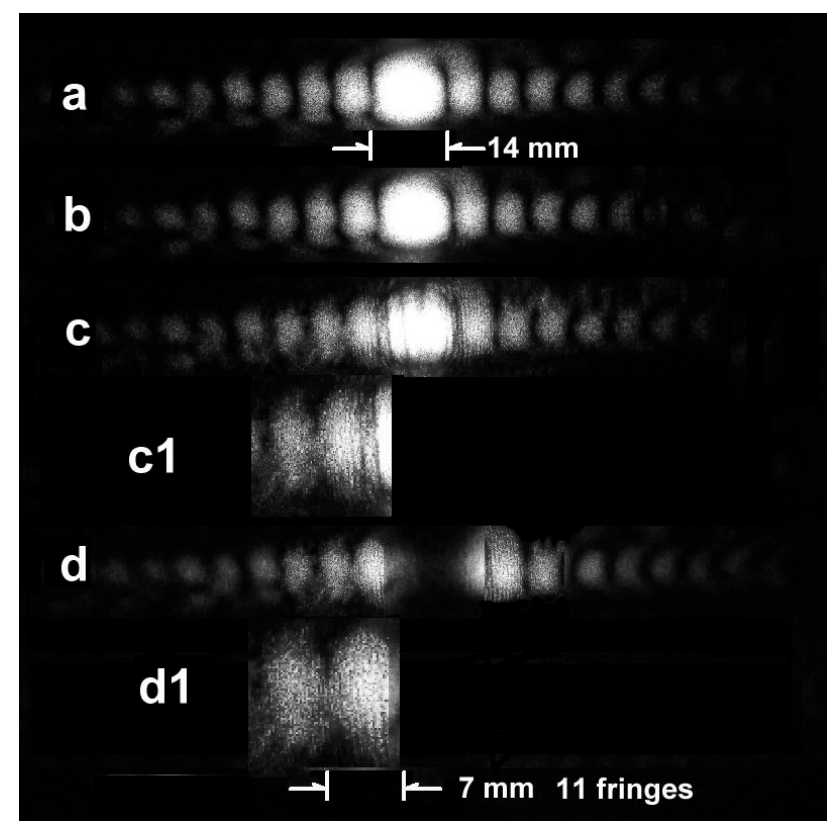

Figure 3. Photographs of the screen images at the experimental stages. 

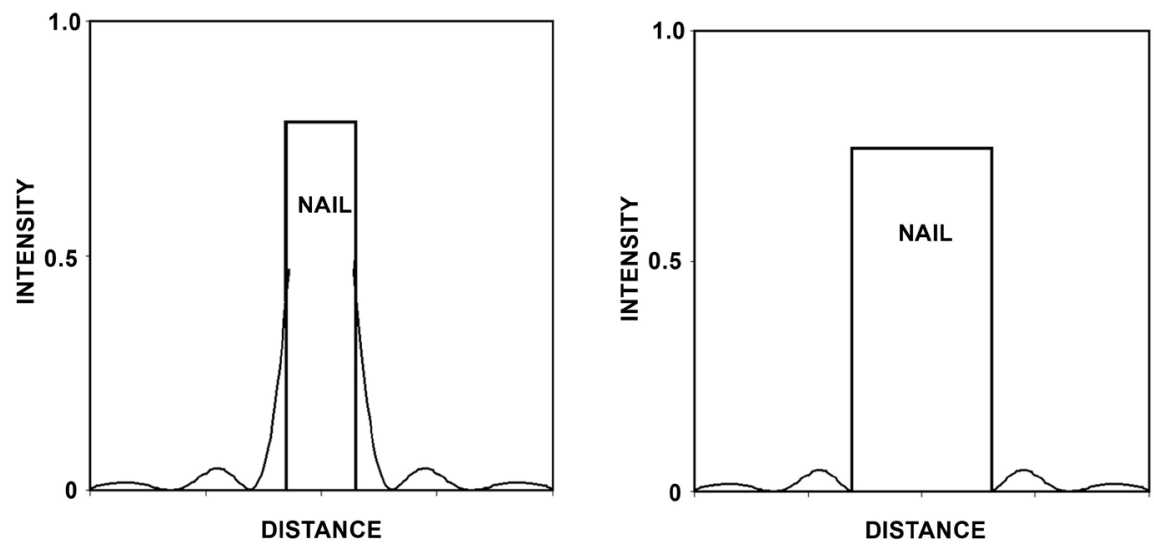

Figure 4. (Left) Opaque strip (the nail) placed in the middle of the primary peak where some of the light in the primary peak passes the nail. (Right) Opaque strip (the nail) placed such that light is blocked from one first minima to the first minima on the other side.
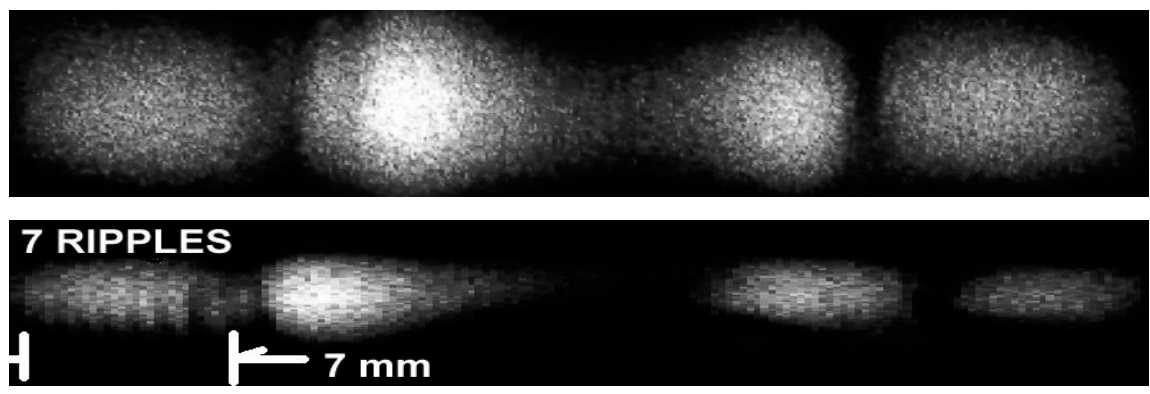

Figure 5. (Top) Opaque strip (the nail) placed as in Figure 4(left). The overall intensity of light is considerably smaller. Interference ripples are present as shown in the image. Note there are ripples behind the opaque strip. (Bottom) Opaque strip (the nail) placed as in Figure 4(right). Note there are NO ripples behind the opaque strip.

the first mask diffraction pattern Figure 4(right). There is a zone with no light behind the nail and interference ripples in the secondary peaks is evident, the bottom of Figure 4.

A nail was positioned to block the central maximum from the first mask between the masks and approximately $94 \mathrm{~cm}$ from the second mask. The nail size was chosen such that its shadow is larger than the slit. The nail was positioned so the edges of the nail correspond to the minima of the central peak as shown in Figure 4(right). The positioning of the blocking nail is critical and difficult to achieve. If it is slightly out of position, some light will be behind the nail. Note the shadow of the nail on the second mask is wider than the slit.

Figure 3(d) is the resulting image on the screen. It is very similar to the interference pattern of Figure 3(c) with the shadow of the nail. Note the interference fringes remain in the second mask image as in Figure 3(c). The images are the same spacing and size. Some nail edge effect can be seen, but the central peak and the light through the slit is blocked. The smaller images Figure $3(\mathrm{c} 1)$ and Figure 3(d1) are expansions of a section of the main images Figure $3(\mathrm{c})$ and Figure 3(d), respectively. The interference fringes in Figure 3(c) and Figure 3 (d) indicate a double-slit experiment. The interference fringes persist after the 
light through the glass slit is blocked.

The last step is to move the glass plates so that the glass plate slit is removed. The image through the glass plate on the screen comes from the blocking nail. This is the bottom of Figure 4. The number of ripples superimposed on the first mask diffraction image in seven $\mathrm{mm}$ has changes from the 11 fringes of the glass plates. The ripples in the first diffraction peak are barely visible. If they were fringes they would be more pronounced.

\section{Discussion and Conclusion}

Instead of the slits being side-by-side as in the traditional Young's experiment, the slits in this experiment are one in front of the other. The glass mask slit modifies each diffraction pattern peak from the first mask to produce the interference pattern on the screen.

Wave models suggest that the waves from the glass mask slit expand and interfere with other waves through the glass mask to form an interference pattern. However, Figure 3(d) image has the light through the second mask slit blocked. The difference between Figure 3(b) and Figure 3(c) suggests that the interference pattern depends on the slit being present and not dependent on light through the slit. That is, the slit is required but light through the slit is not required to form the interference pattern.

This experiment should be repeated with a photon counter.

An experiment was performed with the Fraunhofer pattern from a first mask impinging on a transparent second mask with a slit. The screen pattern was an interference pattern such as produced in Young's Experiment. An opaque strip between the first and second mask blocked the light of the center maxima from the first mask. The screen interference fringes remained. Comparing the placement of the diffraction pattern suggests that the mere presents of the slit induce the fringes. Light through the slit was unnecessary. Moving the glass mask so the slit was removed from light shows the necessity of a slit in the second mask. This suggests a Newtonian type of light model. Because this experiment does not require the Huygens assumptions, this experiment rejects the wave models of light.

\section{Acknowledgements}

André Michaud, Service de Recherche Pedagogiqua Inc., Quebec, QC provided considerable assistance in preparing this paper for publishing.

\section{Conflicts of Interest}

The author declares no conflicts of interest regarding the publication of this paper.

\section{References}

[1] Newton, I. (1952) Opticks. Dover Publications, Inc., New York.

[2] Jenkins, F.A. and White, H.E. (1957) Fundamentals of Optics. McGraw-Hill Book 
Co., Inc., New York, NY, USA.

[3] Hodge, J.C. (2006) Scalar Potential Model of Redshift and Discrete Redshift. New Astronomy, 11, 344-358. https://doi.org/10.1016/j.newast.2005.09.002 